\title{
Post Kidney Transplant Refractory Hypertension and Bilateral Native Nephrectomy
}

\author{
Lerman $\mathbf{M J}$, Hinton $\mathbf{S}$ and Aronoff $\mathbf{R}$ \\ Medical City, Dallas Transplant, Texas, USA
}

\section{Commentary}

Refractory hypertension following kidney or kidney pancreas transplant is a rare but a very serious problem. Most patients can now be successfully and adequately controlled using a combination of calcium blockers, angiotensin converting enzyme inhibitors, angiotensin receptor inhibitors, and diuretics. There is nonetheless a small subgroup of patients who develop severe and sometimes refractory hypertension.

These patients are at significant risk for allograft failure, stroke, congestive heart failure, or even death. We have found that when hypertension is severe and refractory to at least the above combination of medications bilateral native nephrectomy should be considered. It is very important to screen these patients for drug nonadherence, allograft renal artery stenosis, pheochromocytoma, and primary hyperaldosteronism.

We were able to improve blood pressure control in all (6) our patients who met this criteria. Mean arterial blood pressure fell significantly in all patients and the number of antihypertensive medications required to control blood pressure improved in most. Kidney allograft function however remained stable only in our patients with serum creatinine levels less than 2 at the time of nephrectomy. We did not experience any significant complications related to laparoscopic bilateral native nephrectomy in our patients. We believe that patients who developed severe and refractory hypertension which cannot be controlled with medical management should undergo laparoscopic bilateral native nephrectomy as early as possible to preserve allograft function. 